\section{El cuidado del derecho como administración de justicia en la Filosofía del derecho de Hegel}

\author{
The Care of Right as the Administration of Justice in Hegel's Philosophy of Right
}

Pablo Pulgar Moya

\section{RESUMEN}

El presente capítulo tiene como pretensión tanto ilustrar los momentos primordiales del apartado sobre administración de justicia (§ 209-229) al interior de los Lineamientos de la Filosofía del derecho, como exponer algunos elementos conflictivos e n s u comprensión. Este capítulo se separa en tres momentos: i) tematiza la tarea de la administración de justicia como salvaguardia del derecho; ii) caracteriza la cohesión narrativa de la administración de justicia al interior de la sociedad civil y su tensión con el concepto de Estado y, finalmente, iii) la problemática de la p ersonalización al interior del sistema de leyes y la interpelación de Marx. La hipótesis que aparece, entonces, es que Hegel intenta probar la necesidad de la codificación del derecho en virtud la defensa de la libertad personal y de la propiedad, pero la cual solo es posible mediante el poder o violencia judicial.

Palabras claves: administración de justicia; sociedad civil; Estado; personalidad

\section{ABSTRACT}

The aim of this chapter is both to illustrate the main moments of the section on the administration of justice ( $\$ 209-229$ ) within the Lineaments of the Philosophy of Right, and to expose some conflicting elements in its understanding. This chapter is separated into three moments: i) it thematizes the task of the administration of justice as a safeguard of law; ii) it characterizes the narrative cohesion of the administration of justice within civil society and its tension with the concept of the State and, finally, iii) the problematic of personalization within the system of law and the interpellation of Marx. The hypothesis that appears, then, is that Hegel tries to prove the necessity of the codification of law in virtue of the defense of personal freedom and property, but which is only possible through judicial power/violence.

Keywords: administration of justice; civil society; state; personality
INFORMACIÓN

http://doi.org/10.46652/resistances.v2i4.72 ISSN 2737-6222 |

Vol. 2 No. 4, 2021, e21072

Quito, Ecuador

Enviado: noviembre 022021 Aceptado: diciembre 20, 2021 Publicado: diciembre 31, 2021 Publicación continua

Sección dossier | Peer Reviewed

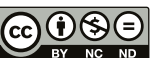

open 2 access

AUTORES

Pablo Pulgar Moya Universidad Católica Raúl Silva Henríquez - Chile

ppulgar@ucsh.cl

Conflicto de intereses

El autor declara que no existe conflicto de interés posible.

Financiamiento

No existió asistencia financiera de partes externas al presente artículo.

Agradecimiento

N/A

Nota

N/A
PUBLISHER 


\section{La administración como cuidado}

El problema de la jurisdicción que trae consigo la noción de sociedad civil hegeliana no comporta pruritos menores al momento de pensar la interrelación entre los estamentos civiles y estatales. La duplicación del análisis, la caracterización de la sociedad civil como distribuidora y administradora de justicia son problemáticas que atañen a la reflexión de la mediación política e instan a la posibilidad de pensar de manera efectiva también al Estado en tanto que pretende mediar los propósitos propios e individuales de las actividades subjetivas. La obertura de los parágrafos sobre la sociedad civil ( $(182-188)$ nos caracteriza la doble lectura de los propósitos perseguidos por los individuos: a) como la realización de los propios intereses egoístas (internamente) y b) como la realización del universal en general a través de la interrelación con otros sujetos en los que consiste (externamente). El carácter lógico de la sociedad civil muestra una macrodialéctica en la que ésta asume el carácter de una eticidad negativa, donde el universal ético exige una mediación entre ambos aspectos. En la ilustración de esa relación es donde aparece la necesidad (Notwendigkeit) de que el sistema de las necesidades (Bedüfnisse) civiles requieran del cuidado y administración de la justicia, a fin de asegurar y resguardar su cumplimiento.

En el presente texto pretendemos dar cuenta de los aspectos principales de lo que Hegel concibe como "administración de justicia” (Rechtspflege), sección medular de la caracterización de la sociedad civil hegeliana y, por tanto, en el corazón de la parte dedicada a la eticidad presentada en su Filosofía del Derecho. La intención principal de esta entrada es describir los pasos fundamentales de la composición del derecho en su marco protector de una propiedad que asegura la personalidad (Persönlichkeit) en y para sí al sistema de necesidades precedente. Es esta "administración de justicia" la que permite, según Hegel, la realización efectiva de la universalidad abstracta de la libertad individual en tanto derecho. Dicho de otra manera, es la Rechtspflege la que asegura, mediante su “aplicación” (Anwendung), que la justicia llegue a instanciarse cuidadosamente según los preceptos de la teoría general del derecho. Esta sección, que compete entre $\S 209$ y $\S 229$ de la obra, articula conceptos fundamentales de la particularidad o particularización del derecho como una ciencia filosófico-jurídica. En nuestra presentación debemos mencionar una dificultad idiomática de entrada, pues el término Rechtspflege enfatiza el cuidado (Pflege), mantenimiento o cargo del derecho (Recht). El cuidado del derecho se vuelve, así, sinónimo de un sustentación, suministro y administración de justicia (Justiz). La predominancia del término alemán por sobre el latino se adscribe a la tarea emprendida en la obra por superar una visión dicotómica entre el derecho natural abstracto y el derecho positivo hipotético enfatizando en la necesidad de ajustamiento constante del derecho en tanto autocuidado jurídico por parte de la sociedad civil, en cuanto ésta administra y le es administrada justicia. El carácter judicativo del derecho muestra su raigambre fenoménica en cuanto es sabida y reconocida por la sociedad civil dotándola de validez universal para la conciencia individual. El derecho abstracto así tendría realidad solo cuando se convierte en ley universal. Esto se menciona al inicio de esta sección: 
Se tiene de esta manera que el pensamiento del derecho tiene que ser constituido para el pensar y no permanecer más en lo meramente sensible; se tiene que adecuar a los objetos la forma de la universalidad (Form der Allgemeinheit) y regirse igualmente en la voluntad según algo universal. Sólo después que los hombres han inventado necesidades (Bedürfnisse) y la adquisición de las mismas se devora en la satisfacción, se pueden constituir leyes (GW 14.1, § 209, Anm.).

La administración de justicia, a veces interpretada como jurisdicción, iuris dictio, manifiesta, primeramente, la mediación entre el derecho con la existencia efectiva (Dasein) a través de ley. Esta ley, sin embargo, no como meramente externa, sino de modo universal (GW 14.1, § 209) viene reconocida, vuelta consciente y requerida, según Hegel, por la sociedad civil en su conjunto. Es menester precisar, sin embargo, que el conocimiento de la regla fundamental componente de la ley en tanto formulación no es suficiente para la consolidación de esta relación, es decir, no se acaba en su aspecto formal, sino en el acabamiento comprehensivo conceptual de su dimensión en la sociedad civil. Este punto no es menor, pues nos da las primeras luces para entender la dimensión redistributiva de justicia en Hegel, como señalara Seelmann (1995, pp. 11 y ss), en tanto Hegel busca entender la pena mediante la aplicación de la ley como una restitución (Widerherstellung) del derecho agraviado. En este sentido, el "cuidado" del derecho que pretende institucionalizar la administración de justicia tiene como tarea primordial el resguardo del universal (derecho) frente a los embates de los individuos, más que un intento de devolver a la senda de "rectitud moral" al sujeto agraviante por medio de la reforma que comporta el ejercicio punitivo. La “administración de justicia” hegeliana enfatiza, entonces, más un carácter de ajustamiento, de repartición de justicia, en razón de una voluntad universal frente a los embates particulares de sujetos racionales, en cuyos propios hombros descansaría su rectitud moral, que la búsqueda de un sistema penal reformador. El cuidado del derecho hegeliano trae consigo, a través de la aplicación ley, la devolución de "dignidad” al derecho de manera universal a la sociedad civil defendiendo la libertad y propiedad de los individuos que son miembros de ésta. Solo a través de este camino el derecho abstracto se vuelve derecho positivo, se reconoce en la sociedad burguesa en su concepto real. En este sentido, el Hegel de la Filosofía del derecho no niega la inmanente contradicción del ejercicio histórico de las costumbres de los individuos, sino que concentra su énfasis en la hegemonía de la sociedad civil, y (solamente) a cierto punto "indiscernible" del Estado, en tanto garante de esa determinación de justicia. Si la sociedad civil encuentra énfasis a las relaciones económicas en el "sistema de las necesidades", es menester enfatizar que se dota también énfasis a las determinaciones jurídicas y políticas de esta misma mediante la "administración de justicia”. Este tránsito, sin embargo, dibuja el intento de Hegel por superar al derecho como expresión abstracta de la libertad del hombre y como expresión de la eticidad del Estado, donde el este último se alzaría como la propia institución legítima surgida al calor de la singularidad civil.

\section{Administración de justicia como centro de discernibilidad entre sociedad civil y Estado.}

La discernibilidad entre sociedad civil y Estado ilustra uno de los puntos más atractivos en el memorable y polémico debate respecto al cariz político de la exposición de la eticidad hegeliana, ora enfatizando en un carácter liberal, ora en uno conservador. Las perspectivas 
contrapuestas disputan el espacio que adquiere la exposición de la sociedad civil, sea bajo un enfoque conservador, de cariz reaccionario vinculado a un fuerte Estado prusiano y rayano a un absolutismo epistemológico-político, por un lado, sea bajo una visión liberal cercana a una matriz proveniente de la economía política inglesa, por otro. Este debate, sin embargo, no es nuevo dentro de la tradición interpretativa de la filosofía del derecho de Hegel, sino que se arrastra desde los mismos contemporáneos a Hegel como Sietze, Schubarth, Stahl, Elsner o Gans, pasando por los comentaristas de la llamada Hegelschule como Ruge, Marx, Rosenkranz o Haym y pensadores tan disímiles como Ritter, Riedel, Foster, Lukács, todos los cuales darán vida a un sabroso debate. Diferentes enfoques que, en cierto punto, se quedará plasmado en las caracterizaciones contemporáneas del pensamiento jurídico, político y ético hegeliano y que encontrará en la aclaración de la administración de la justicia un centro neurálgico.

Como bien advertía Julio de Zan (2012, p. 64), será en las lecciones de Hegel sobre Natur und Staatrecht, del semestre de invierno de 1818, su primer semestre del periodo berlinés, en donde encontraremos recién una diferenciación sistemática entre sociedad civil y Estado, la cual será replicada tanto dos años más tarde en la Filosofía del derecho, como en la tercera edición (1830) de la Enciplopedia. Este elemento será crucial para distinguir la necesidad racional de la relación inmanente entre ambos conceptos. Así:

La sociedad civil de los modernos ya no corresponde, ni en el pensamiento ni en la realidad, a la 'Polis' de los antiguos. Por eso [Hegel] acuñó por primera vez la diferencia conceptual y terminología entre: "sociedad civil” para el sentido moderno, y “Estado" para el sentido clásico y tradicional de la comunidad política. Su esfuerzo estará orientado a distinguir y al mismo tiempo conciliar ambas cosas en su diferencia (De Zan, 2012, p. 64-65).

El término, por tanto, renueva la terminología de la época para dar cuenta de la necesidad de separación ente ambos niveles dotándolo de una carga epistemológica tal que será crucial para el desarrollo del pensamiento político moderno. La caracterización sobre qué constituye a la modernidad, entonces, toma de este aporte de Hegel parte importante para su propia definición. Las esferas de la sociedad civil y la del Estado hasta principios del siglo XVIII se encontraban indiferenciadas adoptando en un mismo ámbito elementos propiamente sociales, políticos, económicos y jurídicos. "En este contexto moderno la teoría hegeliana de la diferencia de las dos esferas se propone rescatar el perdido sentido de lo político” (De Zan, 2012, p. 71), reservando a la sociedad civil su carácter inmanente burgués. El modo de presentación del derecho hegeliano despliega, entonces, un acto de rescate del Estado político que se apropia de la reconstrucción de lo ético, característico de la realización ideal de la “Polis" griega. En este contexto, la administración de justicia se sitúa en el corazón de la estructura de la exposición (Darstellung) de la sociedad civil y funge como gozne entre las necesidades individuales sistematizadas y el binomio administración pública/corporación. La pregunta fundamental, sin embargo, es saber si este cuidado del derecho, esta administración jurídica ilustrada por Hegel, corresponde al modo inmanente de las determinaciones que son necesarias para la construcción conceptual de la sociedad moderna o bien se refiere a un ordenamiento carente de fundamento expositivo. 
El objetivo fundamental de la administración de justicia es cumplir el rol de garante y mediador de los conflictos que puedan surgir entre, por ejemplo, intereses mercantiles entre productores y consumidores, en tanto el derecho subsume universalmente los individuos resolviendo los fácticos y factibles conflictos de interés. No obstante, la problemática se suscita en la derivación conceptual del orden expositivo suministrado por Hegel.

En este punto se encuentran muy de cerca los conceptos de administración de justicia y administración pública (Polizei) lo que se vuelve punto de interés para este segundo acto del presente escrito. Esta unicidad solo es factible a través del reconocimiento civil de la ley en su existencia efectiva. La administración de justicia se alza, de esta manera, como el estadio donde lo universal, el derecho, encuentra realidad efectiva como mediación con lo particular, vale decir, con las necesidades individualizadas.

En el modo de exposición de la idea de derecho se nos va denotando este tránsito, no temporal, sino sistemático, donde el aparataje estatal tiene existencia, a fin de resguardar el mismo derecho, de manera ulterior a la economía política barruntada en el sistema de las necesidades. De esta manera, la Rechtspflege supondría sensu lato el modo expositivo jurídico formal de aquella primacía económica política de los parágrafos inmediatamente anteriores, concretizando los elementos que se manifestaban de manera abstracta. El reconocimiento de la propiedad privada adquiere, ergo, existencia inmediata. La petición organizadora de la sociedad civil llama a resguardar, según Hegel, el derecho a través de "formas de acción” y "diferenciaciones organizacionales" (Blasche \& Schwemmer, 1972, p. 473) de la propiedad privada, dentro de las cuales la administración de justicia tiene preeminencia (GW 14.1, § 208). La estructura estatal moderna en tanto forma-institución arroga para sí la administración de la justicia, pero solo en cuanto su pretensión viene afirmada en el reconocimiento desde la misma sociedad civil, en cuanto ésta se ve enfrentada en la mediación silogística entre lo individual y lo universal, donde la producción de leyes vendría a precaver ese resguardo. La “legítima” violencia (Gewalt) del Estado se transforma, por ende, en razón universal (VRph, 17/18, § 46). La razón como criterio universal de administración de justicia, en tanto cuidado del derecho, encuentra empero una lectura crítica dentro de la misma escuela de jóvenes hegelianos. Arnold Ruge (1840) será quien, primero y con mayor sistematicidad, dote de radicalidad a la crítica a este punto, también Bruno Bauer (1842) y, posteriormente Karl Marx en sus ya conocido Manuscrito de Kreuznach (1843-1844).

Bajo la administración de la justicia de Hegel logra constituirse a modo conceptual el "poder judicial" (richterliche Gewalt), en tanto parte de los poderes del Estado, la cual no tenía una existencia autónoma durante la Edad Media. De esta forma, la implementación “de un poder autónomo de justicia, dentro de la órbita del Estado moderno, requirió, entre otros factores, de la construcción de un discurso legitimador tanto del nuevo Estado-Nación (expresado en la figura del rey) como de la propia actividad de los juristas dentro de él”. (Assalone, 2011, p. 59). El contexto de realización teórica o conceptual (Mertens, 2008, p. 325) de la administración de justicia contiene estos tres momentos, según Hegel, necesarios: 1) derecho como ley (\$ 211-214); 2) Existencia efectiva (Dasein) de la ley (§ 215-218) y 3) el tribunal (§ 219-229). La mediación 
de esas necesidades sistematizadas requiere a la ley como realización y garante del derecho al que corresponde, por lo que en el modo de exposición de esta administración de justicia apremia el reconocimiento de la necesidad del derecho positivo, del derecho en tanto ley o, dicho de otra forma, como derecho puesto (gesetzt). Esta piedra angular remarca el punto de partida para el derecho positivo dentro del sistema de la obra, donde la forma-ley (Gesetzform) encuentra realidad como universal, es decir, como validez sobre los individuos dotada por la misma sociedad civil en el acto de dar realidad efectiva a la disposición racional del sistema de necesidades. En cuanto ley es derecho objetivo en sí, pero que solo lo es en identidad con el ser-puesto (Gesetzsein) (§ 212) y, en esta forma, se comprenderá a sí mismo el derecho no solo como existencia efectiva, sino que tendrá realidad en el mismo proceso de aplicación (Anwendung) de éste. De esta forma se supera la mera forma universal, para traducir el derecho en la particularización que comporta su determinidad, es decir, en el reconocimiento del contenido del derecho en su universalidad determinada (Mertens, 2008, p. 325). Es importante este énfasis del derecho repuesto en la aplicación de la ley, la cual devuelve su carácter de justicia en la sanción penal ante el individuo que ocasiona un daño, no solo a un otro, sino un daño a toda una arquitectura sistémica de necesidades. La reposición cuidadosa del universal jurídico se vuelve entonces, bajo la óptica de Hegel, en necesidad de la misma administración de justicia, en tanto busca ajustar el derecho violentado. Así nos comenta Mizrahi:

En consecuencia, la justificación hegeliana de la pena no funda su legitimidad sobre ningún razonamiento reformista ni de tipo retributivo (como sí lo hacen aquellos de raigambre kantiana) sino sólo sobre la base de uno restitutivo: la pena correspondiente a un delito ha de ser la mínima que permita la restitución (Wiederherstellung) del derecho como tal, es decir, la mínima que suprima al delito qua delito (Mizrahi, 2004, p. 19).

Lo puesto, la ley, será el resguardo del universal ante los propios intereses de las voluntades individuales. La limitación, la sanción, la pena, el castigo, la indemnización serán diferentes medios indispensables en mor de la re-posición (Widerherstellung) del derecho a modo de defensa de los propósitos egoístas. En este sistema:

Siendo puesto y sabido el derecho, se derrumba todo lo accidental del sentimiento, de lo mío, la forma de la venganza, de lo despiadado, del egoísmo, y así primeramente logra el derecho su verdadera determinidad y llega a su honra. Sólo mediante la disciplina del concebir llega [el derecho] a ser capaz de universalidad. (GW 14.1, § 210, An.).

Ahora, sin embargo, será el contenido el que dotará de realidad racional a la ley, una ley que compone a esta sociedad civil/civilizatoria, en la cual la relación material de la forma de universalidad y la individualidad tiene que estar determinada por el contenido que a ella atañe. El contenido de las leyes se implica solo a partir del movimiento sistemático entre derechos y deberes determinados a través de la administración de justicia. El cuidado del derecho, la administración de justicia, indefectiblemente se convierte mediante el ejercicio, mediante la 
aplicación de éste, en coactivo. Para el Hegel de la Filosofía del derecho el derecho administrado es leído como derecho penal, que persigue el control social a través de la sanción punitiva. Esta coacción, en donde el Estado, a través de la administración pública (Polizei), remarca efectivamente su carácter de violencia sobre las partes involucradas en el desacato de la ley universalmente válida para los individuos que toman parte de la sociedad civil. Esta administración trae de vuelta a la sociedad civil a su concepto (GW 14.1, § 229), la cual se había extraviado en la particularidad del derecho abstracto, y la deja predispuesta a la realización efectiva, unitaria, de la administración pública y la corporación, que representan grupos de interés de manera asociativa.

Ahora bien, el derecho no se acaba en la ley formal, sino en cuanto hay una relación vinculante, mediata, entre el individuo y la universalidad de esta ley. En este contexto los individuos se juegan su propia personalidad pues son poseedores de un determinado rol social, de una máscara de personaje dentro de un moderno teatro social. Solo en esa caracterización jurídica, las personas cumplen un rol social donde son igualados al interior de la comunidad. Son personas jurídicas. Esta es la condición de la realización de la libertad consciente de conceptualización de esta conciliación entre individuos y universal (Cobben, 2017, p. 550). El derecho positivo aquí patentado, formulado por Hegel con bastante antelación que el positivismo jurídico, concibe la necesidad de mecanismos punitivos en mor del resguardo del derecho, no obstante, esta pretensión normativa no puede simplemente explicarse de modo redundante, sino que recae en la ley como expresión del derecho, como su exterioridad, suponiendo la universalidad de ésta y la subsunción bajo ella de las voluntades individuales. Será, empero, el respeto mutuo entre los individuos lo que sustentará la realización efectiva de la libertad. El carácter punitivo del derecho, por tanto, no será solo una restricción de la libertad individual, sino su reforzamiento en el marco comunitario frente a otras libertades, libertades pertenecientes a "otras personas". De esta manera, siguiendo a Jakobs, se cumpliría aquí que “el derecho penal como protección de bienes jurídicos significa (ien todo caso!) que una persona, encarnada en sus bienes, es protegida frente a los ataques de otra persona" (Jakobs, 2003, p. 42).

El reconocimiento mutuo de los individuos de la sociedad civil como personalidades, es decir, como personas jurídicas, es una característica de la misma sociedad civil, por lo tanto, antecede (expositivamente) al Estado en cuanto tal. El mutuo reconocimiento como personas dentro de un sistema jurídico, sin embargo, no agota la realización efectiva de la necesidad. Se requiere de la institución.

Sin embargo, esta institución no es meramente formal, ni abstracta, ni anterior a la propia organización, como tampoco lo es la libertad. El Estado moderno hegeliano es y debe ser justo garante y concretización de la libertad sustancial (GW 14.1, § 257), tanto en su carácter subjetivo, como objetivo donde los diferentes momentos de la sociedad civil son subsumidos bajo una universalidad ética, aunque sin sacrificar la libertad subjetiva (Edwards, 1987, p. 27). La cohesión narrativa de la sociedad civil moderna es dotada por la coexistencia entre la realización de la libertad individual y la universalidad del derecho (VRph, 18/19 § 109), la cual en último término es realizada en el aparato del Estado, en tanto que la ley, exigida por la sociedad civil como protección 
de su libre actividad y como garantía de ella misma, no puede desprenderse simplemente de las obligaciones contractuales, sino es producto del Estado. Hegel "la introduce en la sociedad civil mediante la administración de justicia contribuyendo así a configurar la misma sociedad” (Valls Plana, 1997, p. 19). La administración de justicia constituye una coordinación necesaria dentro del marco de la sociedad civil a base del reconocimiento mutuo de una personalidad que se vuelve, en último término, en la base del derecho abstracto, de la propiedad y del contrato.

\section{Personalidad y su conflicto con Marx}

Si bien la administración de justicia puede ser entendida como aparato estatal, lo primordial dentro de la exposición hegeliana es ésta en cuanto funge de garantía del funcionamiento de la satisfacción de las necesidades sistematizadas. No obstante, ella se expone como órgano constitutivo de la sociedad civil, se eleva como gozne mediador entre esta misma sociedad y el Estado, en donde la figura de la ley comienza a adquirir existencia efectiva (Dasein) a fin de darle al tribunal, entre otras cosas, los criterios de juicio concretos para así mediar en caso de conflicto, restaurar al derecho del agravio cometido, sea el autor alguna autoridad gubernamental, sea algún miembro singular de la sociedad civil. El tribunal vendría a pulverizar las asimetrías de poder de los actores que aparecen en la sociedad civil bajo el imperio de la ley del derecho (GW 14.1, § 224; VRph 18/19 § 109, VRph 22/23 § 219, etc.), volviéndose, por tanto, absolutamente necesario como ejercicio de poder público (VRph 18/19 § 109). La voluntad universal adquiere realidad efectiva en el tribunal, en donde todos los integrantes de la sociedad civil se someten, sin dar paso al ejercicio de la venganza privada (GW 14.1, § 221), a la confianza racional del ejercicio del derecho del tribunal como aquel que "imparte justicia”. Será este aspecto represivo institucionalizado de la sociedad civil que se traducirá indefectiblemente en una visión coactiva del Estado, pues por más que la existencia efectiva de la ley miente una perfectibilidad de las costumbres de los miembros de la sociedad, siempre es posible la existencia de la injusticia y la arbitrariedad. El “cuidado" (Pflege), por ende, no aparece en la exposición de Hegel exclusivamente como algo puramente administrativo, sino que también como un carácter de poder/violencia (gewaltig) de lo judicial a modo de reposición del derecho. En su judisdicción, la administración de justicia se presenta en la exposición como una composición negativa resolver los dilemas y conflictos de interés entre "personas" integrantes de la sociedad civil y para dar garantías para la protección del derecho de propiedad y el castigo a su agravio. Este negativo carácter civil, de realización del derecho privado, encuentra una contracara en la administración pública, la cual “en su poder administrativo, tiene la tarea positiva de salvaguardar el orden público y cuidar el bien general, porque sin él el juego libre de intereses privados es imposible" (Wang, 2004, p. 210).

En este respecto, las figuras de la propiedad y el contrato se encuentran formalizadas y reconocidas por la sociedad civil como modos de identificación de los individuos en la red de relaciones sociales expresando aquello bajo códigos y leyes que sustentan la práctica judicial, que emana de una influencia marcadamente kantiana (Kant, 1989 [1797], pp. 47 y 335 ss.). La propiedad, ya tratada como momento constituyente del derecho abstracto como exteriorización de la voluntad, adquiere realidad efectiva en la misma exposición de la sociedad civil en virtud 
del reconocimiento mutuo de sus miembros, que permite la interconexión entre ellos en tanto “personas", es decir, en la medida que detentan un rol social determinado. Nos hemos de enterar en el parágrafo anterior al capítulo dedicado a la administración de justicia (GW 14.1, $\S 208$ ) que la función de ésta, precisamente de sus instituciones, es estar al servicio de la protección del derecho de propiedad en tanto es aquella que le da realidad efectiva al sistema de necesidades, elevando a la sociedad civil a una sociedad regulada, ordenada, la que se entiende en último término como forma de Estado o forma-Estado (Staatform). La sociedad civil forja institucionalidad a modo de ordenar la realización de la idea ética convirtiéndose, como se nos expuso en el sistema de las necesidades en un "Estado del menester y del entendimiento" (Not- und Verstandesstaat) (GW 14.1, § 183). Será entonces esta organización jurídica, como particularidad de la sociedad civil, un paso importante para la caracterización de lo que Xavier Zubiri llamará en Hegel una “ontología del Estado” (1994, p. 225).

La tensión silogística entre lo particular y lo universal que detentan el sujeto y el derecho respectivamente encuentra una concretización mediadora en la administración de justicia y es el lugar donde las determinaciones descritas en el derecho abstracto se realizan mediante el derecho que se ha vuelto positivo, es decir, mediante su existencia efectiva en la ley. El sistema de leyes será el garante del sistema de las necesidades, donde el carácter administrativo del derecho conlleva, de igual manera, un carácter coactivo. El reconocido sujeto de derechos (GW 14.1, § 209) trae consigo un trato a otros sujetos también “como personas" (GW 14.1, § 36), como personas de derechos. La codificación del derecho permite la defensa de la libertad personal y de la propiedad, no obstante, el resguardo de la propiedad es solo posible mediante el poder judicial, en virtud de su violencia "judicativa”. Es la administración de justicia, según Hegel, la que abrirá la puerta para entender a la propiedad como la base sobre la cual los hombres se igualan ante la ley, siendo reconocidos intersubjetivamente de manera universal y proveyendo las instituciones a fin de impedir coactivamente el agravio a la propiedad.

Que el reconocimiento de la personalidad jurídica del individuo venga reconocida mediante la propiedad es un hito de la comprensión moderna del derecho, adquiriendo base legal en mor del requerimiento desprendido desde un sistema de necesidades. La existencia de la persona (la representación del individuo) salvaguarda así el bienestar del individuo (miembro de la sociedad civil) en tanto voluntad particular (Fulda, 2003, p. 220). Esta personalidad jurídica, comprendida por Hegel como principio individuationis, será la que articule, concretice y maximice la libertad del sujeto. Este punto generará conflicto, por otro lado, en la exposición de la dinámica entre el concepto de persona y el de propiedad en la interpelación de Marx. Un primer estadio de conflicto representa la carencia de "personalidad" que comporta la carencia de propiedad privada. Una persona sin propiedad contravendría su rol civil dentro de la sociedad:

Nada puede ser más raro (komisch) que el desarrollo de la propiedad privada de Hegel. El hombre como persona debe dar realidad efectiva a su voluntad como el alma de la naturaleza externa, por lo tanto, tomar posesión de esta naturaleza como su propiedad privada. Si esta es la determinación de 'la persona', el ser humano como 
persona, se deduciría que todo ser humano debe ser propietario de una propiedad para realizarse como persona. Según Hegel, la propiedad privada libre en la tierra, un producto muy moderno, no es una relación social específica, sino más bien una relación del hombre como persona en relación a la 'naturaleza' (MEGA2 II.15, p. 604, n. 26).

A este respecto Marx contraviene el papel en el modo de exposición normativa de la persona al interior de la economía política. La exposición del Marx de El Capital - precisamente en el segundo capítulo - llevará esta reflexión a la interacción al interior de su teoría del valor, es decir, en donde el carácter normativo de la propiedad en el marco el derecho positivo como personificación concreta no vendría ya leída como resultado (expositivo) de la economía política que sustenta el sistema de necesidades, sino como base abstracta (expositiva) integral del proceso de intercambio. Esta reflexión entre dos propietarios es la forma necesaria de subjetividad que media el intercambio (proceso). La personalidad del propietario se basa en una relación legal y está limitada por la forma del contrato componiendo así una relación limitante que sigue un concepto de subjetividad que coordina los miembros de comunidad de modo interdependiente. Este "comportamiento" expresa una confirmación de la independencia personal del propietario, que al mismo tiempo depende objetivamente de su propiedad. El mediato reconocimiento mutuo entre los propietarios conduce a la configuración de la persona como un ser autónomo y aislado sobre la base de un proceso intersubjetivo en donde la persona en tanto propietario tiene poder de comando sobre los objetos. El reconocimiento de la persona (como propietario) es el reconocimiento de su control sobre la naturaleza y esto es al mismo tiempo lo que vuelve plausible al mismo intercambio.

Para Marx, este acto de personificación muestra un cierto fetichismo (Harvey, 2010, p. 47) de la acción humana: la abstención naturalizada y mistificada del ser humano como individuo coincide con el inicio de su acción en la sociedad civil moderna. De esta manera, en la sociedad "burguesa" las personas son fetichizadas deliberadamente: el propietario de un producto posee un objeto susceptible de ser cambiado por otros, por lo que él solo aparece como propietario privado de este producto. Las personas son abstraídas, "fetichizadas" o reducidas a una determinada personificación porque son mutuamente reconocidas como propietarios exclusivos y excluyentes. Esta “fetichización" del propietario como propietario-privado permitiría el comportamiento humano como persona dentro de la red de relaciones modernas. Los sujetos se enfrentan entre sí como representantes de sus posesiones y no reclaman ningún otro contenido epistemológico que el que detentan como personas económico-jurídicas. Este vínculo recíproco del concepto de fetiche y de persona no solo ilustra una relación de sujeto o una corrección de la comprensión cotidiana, sino que retrotrae una crítica directa de este resultado inevitable de la economía política en su conjunto y a una interpelación a estas figuras en la exposición hegeliana de la sociedad civil, incluyendo a la administración de justicia (MEGA2 II.15, p. 604). La tragedia de la "personificación" en la sociedad civil en este "drama” sería justamente la ilusión de la acción libre del sujeto, la cual de hecho no hace más que estar mistificada en la economía política. Lejos de su discurso antropológico de sus primeros escritos, Marx describe 
las relaciones de sujeto bajo relaciones de dependencia socialmente personificadas y formula claramente su ruptura teórica con el supuesto de una conexión social entre la forma civil (o, en Marx, "burguesa”) y una concretización de la libertad personal. La propiedad personaliza al sujeto en virtud de una relación de representación legal, pero lo hace de manera excluyente (en tanto hay sujetos que no podrán representarse al carecer de propiedad), que es lo que le discute a Hegel en su concretización de la persona en la sociedad civil. En otras palabras, los propietarios aparecen en Hegel como personas libres sobre la base de su condición de propietarios privados mutuamente reconocidos y sustentados por el contrato (GW 14.1, § 217), pero que - según Marx - de hecho caen indefectiblemente en la reproducción asimétrica del poder, la que termina siendo resguardada, en último término, por el mismo ejercicio punitivo de la administración de justicia (Pulgar Moya \& Clochec, 2020, p. 29).

\section{Palabras finales.}

Hegel se encuentra embarcado en una feroz discusión con los representantes de la famosa Escuela histórica del derecho, sobre todo ante las concepciones de Savigny y Fries, la cual es patentada durante toda la Filosofía del derecho y la administración de justicia no se excluye de esta disputa. Es también menester tomar en cuenta que la presencia de las formas de la economía política como base social y política dispuestas en el sistema de necesidades da cuerpo a una influencia proveniente ya desde los escritos jenenses. Sería injusto atribuir a Hegel una simple voluntad de reproducción de las figuras económicas liberales muy en boga en su época, pero también es necesario recalcar el entusiasmo y optimismo que tendrá por la construcción del Estado ético, donde la administración de justicia dota a la sociedad civil de instituciones para el resguardo universal del derecho. Por ende, la justicia y lo público siendo administrados se manifiestan como características de la sociedad civil, componiendo una misma intención, pero que encuentran no pocas dificultades en su modo de exposición. La poco representante personalidad jurídica supera una simple interpretación normativa y mienta una reformulación de la universalidad civil del derecho, una "sublevación ante las instituciones", la cual no implicaría "necesariamente la vuelta a una supuesta anarquía original sensu hobbesiano, sino a la reformulación de esas disposiciones y reglas en pro de la justicia” (Cadahia, 2019, p. 226).

\section{Referencias}

Adorno, T. (1970). Negative Dialektik. Suhrkamp.

Assalone, E. (2011). Hegel y la fundamentación burocrática de la filosofía. La relación entre la filosofía y el Estado en los Principios de la Filosofía del Derecho de Hegel. Revista Filosofía UIS, 10(2), 52-76. https://revistas.uis.edu.co/index.php/revistafilosofiauis/article/view/2834

Bauer, B. (1842). Hegels Lehre von der Religion und Kunst von dem Standpuncte des Glaubens aus beurtheilt. Otto Wigand.

Blasche, S. \& Schwemmer, O. (1972). Methode und Dialektik. Vorschläge zu einer methodischen Rekonstruktion Hegelscher Dialektik. In M. Riedel. (ed.). Rehabilitierung der praktischen Philosophie. Rombrach. 
Cadahia, L. (2019). Del mundo de la utilidad a la experiencia de la libertad: Hegel y la política de nuestro tiempo. En F. Duque. (ed.). Hegel. Lógica y constitución. (pp. 197-226) Círculo de Bellas Artes.

Cobben, P. (2017). Hegel's Concept of Corporation as the Mediation between Free Market and State, Filozofija I Društvo, XXVIII (3), 543-559. https://doi.org/10.2298/FID1703543C

De Zan, J. (2004). La ética, los derechos y la justicia. Konrad Adenauer Stiftung.

De Zan, J. (2012). Hegel y América. La interpretación hegeliana de la dialéctica sociedad civil-estado en Europa y América, América, 21, 59-94

Edwards, P. (1987). Positive Law in Hegel's Philosophy of Right, Sigma: Journal of Political and International Studies, 5 1-1. https://scholarsarchive.byu.edu/sigma/vol5/iss1/3

Fulda, H. (2003). G. W. F. Hegel. Beck Reihe

Harvey, D. (2010). A companion to Marx’s Capital. Verso.

Hegel, G. W. F. (VRph) (1974). Vorlesungen über Rechtsphilosophie 1818-1831. 6 vols. Ilting, K. (ed.). Frommann-Holzboog.

Hegel, G. W. F. (GW 14.1) (2009). Grundlinien der Philosophie des Rechts. In K. Grotsch \& E. Weisser-Lohmann. Gesammelte Werke Vol. 14.1. Felix Meiner Verlag

Jakobs, G. (2003). El funcionalismo en el Derecho penal. Libro homenaje al profesor Günther Jakobs. Universidad Externado de Colombia.

Kant, I. (1989). La Metafísica de las costumbres. Tecnos.

Marx, K. (2004) (MEGA2 II.15). Karl Marx - Das Kapital. Kritik der politischen Ökonomie Erster Band, Hamburg - 1872. In Marx \& Engels Gesamtausgabe (MEGA). Dietz Verlag.

Mertens, S. (2008). Die juridische Vermittlung des Sozialen: die konzeptuelle Basis der reifen Theorie des Juridischen und die Bedeutung der Theorie des Rechts für die Theorie des komplementären Zusammenhanges von Gemeinschaft und Gesellschaft moderner sittlicher Gemeinwesen in G.W.F. Hegels 'Grundlinien der Philosophie des Rechts'. Könighausen u. Neumann.

Mizrahi, E. (2004). La legitimación hegeliana de la pena. Revista De Filosofía, 29(1), 7-31. https://revistas. ucm.es/index.php/RESF/article/view/RESFo404120007A

Pulgar Moya, P., \& Clochec, P. (2020). Límites y desarrollo del concepto de sociedad en Marx y la herencia terminológica desde Hegel. Hybris. Revista de Filosofía, 11(1), 13-43. http://revistas.cenaltes.cl/index. php/hybris/article/view/327

Ruge, A. (1840). Politik und Philosophie. Hallische Jahrbücher Nr. 292 y 293, 5 y 7 diciembre.

Seelmann, K. (1995). Hegels Straftheorie in seinen Grundlinien der Philosophie des Rechts. In K. Seelmann. (ed.). Anerkennungsverlust und Selbstbehauptung: Hegels Straftheorie.

Valls Plana, R. (1997). Sociedad civil y Estado en la Filosofía del derecho, Tópicos. Revista de Filosofía de Santa Fe, 5, 3-27. https://doi.org/10.14409/topicos.voi5.7371 
Wang, Z. (2004). Freiheit und Sittlichkeit. Königshausen \& Neumann.

Zubiri, X. (1994). Naturaleza, historia, Dios. Alianza/Fundación Xavier Zubiri.

\begin{abstract}
AUTOR
Pablo Pulgar Moya. Doctor en Filosofía por la Ruprecht-Karls-Universität Heidelberg; Licenciado en Educación en Filosofía por la Pontificia Universidad Católica de Valparaíso. Profesor Adjunto del Instituto de Filosofía de la Universidad Católica Silva Henríquez. Presidente de la Sociedad Iberoamericana de Estudios Hegelianos.
\end{abstract}

